\title{
THERAPY
}

\section{Targeting chromatin remodelling proteins to treat pancreatic cancer}

Targeting chromatin remodelling proteins is an effective way of suppressing pancreatic ductal adenocarcinoma (PDAC) in both in vitro and in vivo models, according to a new paper published in Nature Medicine.

Next-generation sequencing data has previously shown frequent alterations to genes that regulate chromatin remodelling in human PDAC tumours, and so the researchers investigated whether epigenetic inhibitors of this process could be used to treat PDAC. Initial focus centred on the compound JQ1, an inhibitor of bromodomain and extraterminal family proteins that recognize acetylated lysine in histones and induce transcription of oncogenes such as MYC and inflammatory cytokines. JQ1 treatment was compared with the standard-ofcare chemotherapy, gemcitabine, in a mouse model of PDAC. JQ1 reduced MRI-measured tumour volume and increased survival, whereas gemcitabine had little effect on these outcomes. "JQ1 works by inhibiting the activity of the MYC oncogene, but also by blocking inflammatory molecules," says corresponding author Jens Siveke. The tumours of JQ1-treated

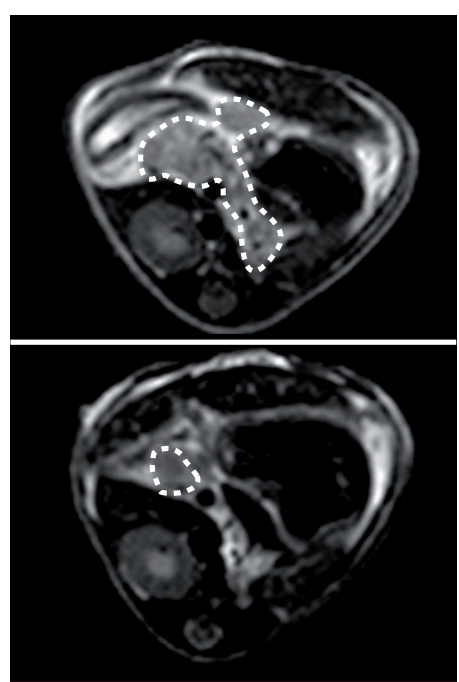

PDAC tumour area before (top) and after (bottom) JQ1 + vorinostat therapy. Courtesy of J. Siveke. mice had reduced expression of Myc targets and reduced serum concentrations of inflammatory cytokines (Il-6 and Il-1 $\alpha$ ), both of which exacerbate pancreatic tumorigenesis. Injections of IL-6 were found to block the therapeutic benefit of JQ1.

As PDAC was eventually lethal to mice treated with JQ1 alone, the investigators combined JQ1 treatment with an FDA-approved HDAC inhibitor, vorinostat. In mice with PDAC, the combination therapy increased median survival time from 24 days with JQ1 monotherapy to 47 days, with no evidence of systemic toxicity. Importantly, this increase in survival time was synergistic, greater than the additive effect of combining both drugs.

Analysis of the gene expression data in PDAC tumours from mice and humans revealed that the tumour suppressor gene CDKN1C (also known as p57) was the most synergistically upregulated gene during combination treatment.

The authors hypothesized that CDKN1C was contributing to the effects of JQ1 and vorinostat treatment, and so they deleted pancreatic Cdkn1c in adult mice using CRISPR-Cas9 technology. Tumours with successful deletion of $C d k n 1 c$ showed substantially reduced apoptosis compared with those that retained Cdkn1c expression.

"We need to determine if this treatment is a combination strategy that is specific to $R A S$-driven tumors or if it may work for other cancers-it'd be great if this strategy worked on many tumour types!" exclaims corresponding author Julien Sage.

Hugh Thomas

Original article Mazur, P. K. et al. Combined inhibition of BET family proteins and histone deacetylases as a potential epigenetics-based therapy for pancreatic ductal adenocarcinoma. Nat. Med. doi:10.1038/nm.3952 